\title{
Le Poète, le Philosophe et le Savant-Écrivain
}

Q uelques mots sur trois rencontres heureuses...

La recherche littéraire est une aventure incertaine, faite de déplacements, de privations, de dur travail mental et physique, aboutissant parfois à des résultats parcimonieux - ingratement reçus aussi! - parfois donnant sur l'introuvable et donc sur la vanité de l'effort et la peine de l'échec. Quelle joie donc de faire l'expérience de deux moments propices, fructueux, et cela sans beaucoup d'effort et, pour ainsi dire, dans l'arrière jardin de chez soi.

Les neuf lettres inédites de Paul Claudel à Étienne Gilson, je les ai découvertes dans les archives de l'Institut Pontifical Médiéval au Collège Saint Michaels de l'Université de Toronto, grâce à l'aide généreuse de l'archiviste, le père Fred Black. Elles sont publiées ici avec la permission de l'Institut Pontifical et de Madame Claudel-Nantet, fille de Paul Claudel. Ces lettres ${ }^{1}$ nous donnent des aperçus de l'homme intime: sa générosité, sa droiture et même son humour; encore d'autres indices sur le poète: ses valeurs littéraires et religieuses, - l'importance qu'il attribue à Saint Thomas mais aussi à Saint Bonaventure; et des aspects de la vie du diplomate, ambassadeur à Washington et à Bruxelles. Dans les années vingt, le philosophe Alain devint l'exégète de La Jeune Parque de Valéry. Gilson ne fit rien de comparable pour la poésie de Claudel. Pourtant il en était un grand admirateur et il ne cessa d'en parler au cours de ses nombreuses conférences dans plusieurs universités nord-américaines. Les lettres ci-dessous sont encore un témoignage de leur fraternité spirituelle et intellectuelle.

L'essai inédit de Gilson, "Digressions sur l'objet de l'expérience poétique," vient aussi de cette riche mine qui est les archives de l'Institut Pontifical Médiéval dont Gilson a été l'un des fondateurs. Le penseur, qui a écrit longuement sur l'esthétique et les beaux-arts, médite ici l'idée de la poésie pure, ce qui fut l'objet d'un long débat parmi les intellectuels français des années vingt. Gilson s'acharne dans cet essai à élucider la différence entre prose et poésie. Jamais l'impossible ne devint si simple! On y admire une lucidité sans pareil: le mariage parfait de la pensée et de sa forme d'expression, sans aucune nuance de l'obscur ni du complexe. Cet essai égale en profondeur et en virtuosité stylistique une autre merveille du genre, celui de 
Valéry, "Poésie et pensée abstraite." C'est encore une défense et illustration du lien fondamental entre la poésie et la philosophie.

Dans cette même veine, il faut placer la rencontre de Gaëtan Brulotte que j'accueille avec grand plaisir dans l'équipe de rédaction de LittéRéalité. Ce "savant-écrivain" partage sa vie entre ses activités de professeur à l'Université de la Floride du Sud, à Tampa, et son engagement comme écrivain, surtout dans son Québec natal. Gaëtan Brulotte exemplifie depuis plusieurs années un talent fertile et novateur. II s'est distingué dans les lettres québécoises surtout pour l'élan qu'il a redonné à la narration et en particulier à la nouvelle. Il est l'auteur de plusieurs livres de fictions dont L'emprise (1989), Le surveillant (1982), Le client (1983) et Ce qui nous tient (1988). Cet ancien étudiant de Roland Barthes a aussi signé plusieurs études de critique littéraire. Sa dernière publication, L'Univers de Jean-Paul Lemieux ${ }^{2}$ a été accueilli par la critique avec une ovation générale: "une étude sérieuse, captivante, sensible et accessible," dit Serge Bruneau à Radio Canada; et un ouvrage "aussi substantiel que novateur," affirme Clément Marchand de la Société Royale du Canada.

Quelle chance d'avoir Gaëtan Brulotte avec nous! Sa contribution va certainement marquer et enrichir notre revue. Parmi ses responsabilités, il va nous aider à donner une présence plus ample et plus étoffée à la littérature québécoise dans LittéRéalité. Nous espérons ainsi ajouter un peu de notre énergie à faire mieux connaître la littérature contemporaine du Québec dans des régions francophones et anglophones; et par ce lien aussi mieux diffuser LittéRéalité au Québec. Bienvenue, Gaëtan!

Sergio Villani

\section{Notes}

${ }^{1}$ Voir à ce sujet, Sergio Villani, "Étienne Gilson: lecteur-critique de Paul Claudel," Claudel Studies Vol XXIV, Number 1 \& 2 (1997): 65-71.

${ }^{2}$ Gaëtan Brulotte, L'Univers de Jean-Paul Lemieux (Montréal: Fides, 1996). 